\title{
RESONANCE OF MIXING ENERGY AND ENERGY OF ELASTIC DEFORMATIONS DURING SPINODAL DECOMPOSITION AND THE COMPOSITION MODULATION EFFECT IN $\mathrm{Zn}_{x} \mathrm{Cd}_{1-\mathrm{x}}$ Te SOLID SOLUTIONS
}

\author{
Pavel Moskvin ${ }^{1}$, Sergii Skurativskyi ${ }^{1}$, Wojciech Sadowski ${ }^{2}$, Barbara Koscielska ${ }^{2}$, \\ Petro Melnychuk ${ }^{l}$, Oleksandr Prylypko ${ }^{1 *}$ \\ ${ }^{I}$ Zhytomyr Polytechnic State University, 103 Chudnivska str., Zhytomyr 10005, Ukraine \\ ${ }^{2}$ Faculty of Applied Physics and Mathematics, Department of Solid State Physics, \\ Gdansk University of Technology, Gabriela Narutowicza 11/12, 80-233 Gdansk, \\ Poland
}

Received 27.01.2021

Accepted 30.06.2021

\begin{abstract}
The Cahn-Hilliard equation is adapted to consider the spinodal decomposition of $\mathrm{A}^{2} \mathrm{~B}^{6}$ semiconductor solid solutions. This approach is used to analyze the process of spinodal decomposition of $\mathrm{Zn}_{\mathrm{x}} \mathrm{Cd}_{1-\mathrm{x}} \mathrm{Te}$ solid solution, which is accompanied by the appearance of the composition modulation effect during its low-temperature synthesis. Numerical simulations of the spinodal decomposition of the $\mathrm{Zn}_{\mathrm{x}} \mathrm{Cd}_{1-\mathrm{x}} \mathrm{Te}$ solid solution are performed. It is shown that micro-variations of the material composition are related by the resonance phenomenon between the excess mixing energy and the energy of elastic strains arising in the inclusions of the new phase, which are coherently conjugated with the initial crystal lattice. It is revealed that such resonance phenomena are most intense when the conditions for the material synthesis are located in close proximity to the spinodal curves on the phase state diagram of the system.
\end{abstract}

Keywords: semiconducting II-VI materials; phase equilibria; spinodal decomposition.

${ }^{*}$ Corresponding author: Oleksandr Prylypko,poizh77@gmail.com 


\section{Introduction}

$\mathrm{A}^{2} \mathrm{~B}^{6}$ semiconductor solid solutions are of practical interest for modern electromagnetic radiation recorders on a wide range of wavelengths. Modern technological methods for synthesizing superthin (nano) layers of this class of materials are usually carried out at sufficiently low temperatures [1-4]. It is the use of low temperatures that determines the possibility of obtaining thin layers of high chemical purity with high crystal structural perfection. At the same time, low synthesis temperatures mean that the thermodynamic state of the $\mathrm{Zn}_{\mathrm{x}} \mathrm{Cd}_{1-\mathrm{x}} \mathrm{Te}$ solid solution is close to the state of its spinodal decomposition. This statement follows from experimental and theoretical studies of the low-temperature part of the $\mathrm{Zn}$-Cd-Te system phase diagram [3-4], where it is shown that the critical temperature of the spinodal decomposition of the solid phase is at the level of $300^{\circ} \mathrm{C}$. It points out that under typical conditions of a technological process, the synthesis of layers of this system, for example, by vacuum technologies [1,2], is performed at lower temperatures on the substrate. This situation leads to additional studies of thermodynamic processes occurring during phase formation in these semiconductor systems and under such conditions.

An essential consequence of the supersaturated state of the solid phase formed under spinodal decomposition is the theory-predicted modulation effect of the composition of the solid solution [5-7]. The essence of this effect is the formations in the solid solution lattice the micro oscillations of its composition with the formation of a kind of superstructure. The transition to a stable state of such a supersaturated solution, as a rule, is accompanied by the appearance of new phases, the crystal lattice period (CLP) of which differs from the CLP of the matrix of the starting material. The formation of such new precipitates while maintaining coherent conjugation of the periods of the lattices of the interconnected phases, in turn, involves energy costs for the formation of new elastically deformed states in the initial crystal lattice.

We could not find in the literature direct experimental results on the detection of micro oscillations of the composition in the layers of $\mathrm{A}^{2} \mathrm{~B}^{6}$ solid solutions or, what is the same, on the composition modulation effect, in layers that were grown under thermodynamic conditions close to boundaries of the spinodal decompositions. At the same time, for a number of $\mathrm{A}^{3} \mathrm{~B}^{5}$ semiconductors solid solutions synthesized, as a rule, at sufficiently low temperatures, this effect was observed experimentally [8-10]. It should be noted that the micro oscillations of the composition of the solid solutions are found in epitaxial layers, which were obtained by fundamentally different methods, both from the gas and liquid phases. Thus, by means of transmission electron microscopy, it was possible to detect periodic changes in the composition in the epitaxial layers both in $\mathrm{Ga}_{\mathrm{x}} \mathrm{In}_{1-\mathrm{x}} \mathrm{P}$, ternary solid solutions grown on a GaAs substrate [8] and quaternary $\mathrm{Ga}_{\mathrm{x}} \mathrm{In}_{1-}$ ${ }_{x} \mathrm{P}_{\mathrm{y}} \mathrm{As}_{1-\mathrm{y}}$ systems grown on InP substrates [9-10]. It has been established that metastable solid solutions grown at temperatures below $T_{c}$ are characterized by reduced mobility of charge carriers due to their scattering in homogeneities of composition. An increase in the epitaxy temperature in such systems eliminates spinodal decomposition on the growth surface, which favorably affects the electrical properties of the epitaxial layers [9].

These studies were carried out on heterostructures, where elastic deformations in the layers arose when their CLP deviated from the lattice period of the massive substrate. Such an elastically deformed state of inclusions of the precipitated new solid 
phase was preserved, naturally, as long as coherent conjugation of the crystal lattices in the heterostructure remained. It was this that created the possibility of the appearance of an oscillatory process associated with the transition of the energy of a decaying solid phase into the elastic energy of deformed inclusions. Such a scheme of spinodal decomposition of the material was analyzed by us in [11] as applied to the description of the composition modulation effect in a solid solution forming a $\mathrm{Ga}_{\mathrm{x}} \mathrm{In}_{1-\mathrm{x}} \mathrm{P}-\mathrm{GaAs}$ heterostructure.

At the same time, in modern synthesis methods, layers of a solid solution are often deposited on an amorphous substrate (glass) or are obtained in the form of a polycrystalline structure. It was under such conditions that the layers of $\mathrm{Zn}_{\mathrm{x}} \mathrm{Cd}_{1-\mathrm{x}} \mathrm{Te}$ solid solutions were obtained and studied by us in $[1,2]$. In such a situation, the inclusion of a new solid phase that appears due to the decomposition process differs in the CLP from the average CLP of the main matrix of the solid solution. This leads to a different method for calculating the values of elastic strains in the system, in comparison with the technique used in [11] when the elastic state of the film was controlled by the crystallographic parameters of the massive substrate. Indeed, in the case of the formation of an elastically deformed inclusion in the crystalline matrix of the material, its excess elastic energy must be calculated concerning the CLP of the initial material itself. The latter, naturally, should also be reflected in the results of modeling the process of spinodal decomposition of a material. The foregoing suggests additional theoretical studies of the spinodal decomposition of $\mathrm{A}^{2} \mathrm{~B}^{6}$ semiconductor solid solutions, which are synthesized by the indicated technological methods and are characterized by different conditions for the appearance of the elastic energy component in the system.

In this article, it is presented in full the thermodynamic and mathematical model for the spinodal decomposition of $\mathrm{A}^{2} \mathrm{~B}^{6}$ ternary semiconductor solid solutions when the deposition occurs without the stabilizing effect of a massive substrate. The findings obtained refine and supplement the results presented in the paper [12].

\section{Thermodynamics of spinodal decomposition}

In [11], the fundamental equations for a spinodal decomposition of Cahn and Hilliard [5-6] were adapted to describe spinodal phase transformations occurring in the decay of a $\mathrm{Ga}_{\mathrm{x}} \mathrm{In}_{1-\mathrm{x}} \mathrm{P}$ semiconductor solid solution grown on a GaAs substrate. In the present work, this approach is extended to describe the process of spinodal decomposition of $\mathrm{Zn}_{\mathrm{x}} \mathrm{Cd}_{1-\mathrm{x}} \mathrm{Te}$ solid solution when its layers or polycrystallites undergo spinodal decomposition in the absence of external stabilizing action of the substrate. When modeling the spinodal decomposition process for $\mathrm{Zn}_{\mathrm{x}} \mathrm{Cd}_{1-\mathrm{x}} \mathrm{Te}$ solid solutions in a wide range of its compositions are perform, the CLP of the solid phase matrix must be considered as a current variable related to the average composition of the material. This is a fundamental difference between the approach of the present work and the conditions for decay simulation when the CLP of the deposited film is stabilized by the CLP of the substrate.

To derive the equation for the kinetics of spinodal decomposition, we start from the balance law for the concentration $c$ written in the classical form $\rho c_{t}=-\nabla J$, where the flux $J \sim \nabla W$. In turn, the potential (free energy) $W=G^{e x}+G^{e l}-\beta \nabla^{2} c$, where the terms $G^{e x}$ and $G^{e x}$ correspond to excess molar energy of solid phase mixture and 
excess phase energy of the deformed crystal (the expressions for them are given in [11]), the last term describes the dependence of $W$ on the gradient of composition, $\beta$ is the coefficient of power series for the free energy corresponding to the solid solution which is heterogeneous over its composition [5-7, 11]. Since we are interested in the steady processes, the above equation follows the relation $G^{e x}+G^{e l}-\beta \nabla^{2} c=\mu=$ const , where the parameter ${ }^{\mu}$ coincides with the chemical potential of the solid phase [5-7,11] and is related to the mass conservation law [7]. Thus, the final expression for the spinodal decomposition equation reads as follows:

$$
\beta^{2} x / d z^{2}=\alpha^{S} x \cdot(1-x)+R T[x \ln x+(1-x) \ln (1-x)]+\lambda_{i j k} \cdot N_{O} a\left(a-a_{S}\right)^{2} / 4-\mu=F(x),
$$

where $z$ is the coordinate in the direction of layer growth; $x$ is the molar fraction of $\mathrm{ZnTe}$ in the $\mathrm{Zn}_{\mathrm{x}} \mathrm{Cd}_{1-\mathrm{x}} \mathrm{Te}$ solid solution; $\alpha S$ is the solid-phase interaction parameter in the model of regular solutions for the ternary solid phase $\mathrm{Zn}_{\mathrm{x}} \mathrm{Cd}_{1-\mathrm{x}} \mathrm{Te} ; N_{O}$ is the Avogadro number; $a, a_{s}$ are the current and average CLP layer (lattice period of the initial solid solution matrix); $\lambda_{i j k}$ - the combination of the elastic moduli $C_{i j}[11,13]$; $R=8.31 \mathrm{~J} /(\mathrm{mol} \mathrm{K})$ is the gas constant, $T$ is the absolute temperature.

In this work, we simulate the composition modulation effect in the $\mathrm{Zn}_{\mathrm{x}} \mathrm{Cd}_{1-\mathrm{x}} \mathrm{Te}$ solid solutions layers in the crystallographic direction $\langle 111\rangle$. Therefore, the following expression for calculating the energy parameter for the indicated crystallographic orientation was used [11]:

$$
\lambda_{111}=\frac{6 C_{44}\left(C_{11}+2 C_{12}\right)}{C_{11}+2 C_{12}+4 C_{44}},
$$

where $C_{i j}$ are the elastic constants of the solid solution.

The problem turns out to be fully formulated when we take into account the ratio for the average concentration of the component in the solid solution:

$$
\bar{x}=\frac{1}{L} \cdot \int_{0}^{L} x(z) d z,
$$

where $L$ is the period of composition oscillations.

The final statement of the problem of calculating the distribution of the solid solution composition over the thickness of the grown layer involves the selection of initial conditions for the decomposition process. It is the choice of the initial content of the component in the solid solution that allows you to set the concentration interval in which the search for solutions to the initial value problem will be carried out. The classical conditions for this problem are the following:

$$
x_{\text {init }}=\text { const }, \frac{d x}{d z}_{z=0}=0 .
$$


Thus, differential eq. (1) with initial conditions (3) and substance conservation condition (2) forms an integro-differential nonlinear problem that fully characterizes the distribution of the composition of semiconductor solid solutions of class $\mathrm{A}^{2} \mathrm{~B}^{6}$, which are synthesized under thermodynamic conditions close to the spinodal decomposition boundary of the material and in the absence of an external stabilizing effect of the substrate.

\section{Thermodynamic parameters and elastic properties of the Zn-Cd-Te system.}

The distribution of the components over the layer of the solid solution after its spinodal decomposition is determined by the excess energy of mixing of the components in the solid phase and the elastic parameters of the crystal matrix of the solid solution, i.e. elastic constants $C_{i j}$. The elastic constants required for the simulation, as well as the data on CLP for semiconductor compounds forming ternary $\mathrm{Zn}_{\mathrm{x}} \mathrm{Cd}_{1-\mathrm{x}} \mathrm{Te}$ solid solutions, are presented in Table 1. The elastic properties of the solid solution, as its CLP, for each current composition were modeled using linear approximations for each of the material parameters according to traditional expressions [11].

Table 1. Elastic constants and crystal lattice parameters of CdTe and ZnTe compounds at $T=298 \mathrm{~K}$ [12].

\begin{tabular}{lllll}
\hline Compound & $C_{11}, \mathrm{hPa}$ & $C_{12}, \mathrm{hPa}$ & $C_{44}, \mathrm{hPa}$ & CLP, $\mathrm{nm}$ \\
\hline CdTe & 53.8 & 37.4 & 20.18 & 0.648 \\
ZnTe & 72.2 & 40.9 & 30.80 & 0.608 \\
\hline
\end{tabular}

An important fragment in solving the problem is to take into account the temperature dependences of all parameters that are necessary for the implementation of the model. At the same time, the available data on their temperature changes show that the variations in these values during the transition from typical synthesis temperatures to room temperature do not exceed several percent (Table 1). Therefore, taking into account the existing inaccuracy in both the experimental determination of the magnitude of the parameters themselves and their temperature coefficients, it was decided to neglect the change in the quantities under consideration and to use in the analysis their values related to the room temperature.

Fundamentally important is the choice of the parameter of the solid-phase interaction between the main components of the $\mathrm{Zn}_{\mathrm{x}} \mathrm{Cd}_{1-\mathrm{x}} \mathrm{Te}$ solid solution. Indeed, this parameter characterizing the excess energy of mixing of the components in a solid solution not only used when describing the composition distribution in eq. (1), but its value is closely related to the critical temperature of spinodal decomposition and the position of boundaries of absolutely unstable and metastable regions on the temperature-composition phase diagram. The position of the indicated boundaries in their essence sets the intervals of thermodynamic parameters, where the application of the analysis performed is justified, and its results are most informative.

As a rule, the indicated parameter and its temperature dependence are found by processing the data on phase equilibria in the systems in accordance with the model of simple solutions in different approximations. Despite the limited experimental information on the state diagrams of $\mathrm{Zn}-\mathrm{Cd}-\mathrm{Te}$ systems, especially at the low- 
temperature interval, the solid-state interaction parameters reported in the literature do not contradict their physical meaning and correlate well with each other for $\mathrm{A}^{2} \mathrm{~B}^{6}$ systems $[4,12]$. Such a situation justifies the possibility of using the data from works [4, 12] for modeling the process of spinodal decomposition of the solid solutions to be considered. The above considerations allow us to take the following value of the parameter in the calculations $\alpha_{\text {ZnTe-CdTe }}^{S}=(17230-14.83 \cdot T) \mathrm{J} / \mathrm{mol}$. The critical decomposition temperature corresponding to the accepted interaction parameter was $547 \mathrm{~K}$. In the calculations, we used the relationship between the interaction parameter and critical temperature known from the simple solutions theory [4]: $T_{c}=\alpha^{S} /(2 R)$. The results of calculations of the critical temperature clearly confirm the statement made earlier that for $\mathrm{Zn}_{\mathrm{x}} \mathrm{Cd}_{1-\mathrm{x}} \mathrm{Te}$ solid solutions, the thermodynamic conditions of layer growth turn out to be very close to the boundaries of unstable states of the solid phase of the material.

\section{The mathematical software for the spinodal decomposition problem}

Eq. (1), which describes the distribution of the composition of the solid phase, the thermodynamic state of which is located near the boundary of the spinodal decomposition, is a second-order nonlinear differential equation. The nonlinear nature of the equation is due to complex relationships between the thermodynamic and elastic properties of the crystal lattice with the solid solution composition.

The search for thermodynamic conditions when oscillation transitions of the excess energy of mixing a solid solution into the energy of elastic deformations of inclusions of a new phase appear in the system was carried out in accordance with the procedure from [11]. For this, the phase portrait of differential eq. (1) was analyzed. The expression for constructing such dependence was obtained by analogy with [7], and for the equation, in question, the desired derivative can be calculated by the formula given in [11].

In accordance with this expression and with selected constants $\beta$ and $\mu$ one can obtain quantitative information about the behavior of the desired derivative. In the problem of finding the intervals of system parameters where periodic solutions of the differential equation under consideration will be observed, it is sufficient to qualitatively track these dependencies. Therefore, the specific values of these constants at this stage of the analysis do not carry informational values. In such a situation, to simplify the calculations, it seems appropriate to use $\beta=1$ and judge the course of the dependence of the derivative accordingly on composition in dimensionless form. Naturally, the composition of the solid solution was chosen as an independent variable in the analysis. Such a choice of coordinate axes also meets the requirements for constructing a phase portrait for the differential equation in question.

A typical view of the phase portrait of eq. (1) derived at the temperature of $450 \mathrm{~K}$ is shown in Figure 1. It should be noted that the chosen calculation temperature is obviously lower than the critical temperature $(547 \mathrm{~K})$ of the spinodal decomposition of the material. 
As in [11], the singular points of eq. (1) when $F\left(C_{1}\right)=0$ and $F\left(C_{2}\right)=0$ are marked by symbols $C_{1}$ and $C_{2}$. Naturally, the parameter forming the set of level lines (isoenergetic lines) in Figure 1 is the total energy of a system $E=\beta(d x / d z)^{2}-2 \int_{C_{1}}^{x} F(s) d s$.

Figure 1 shows the existence of such phase trajectories at the expected values of the initial parameters of the system. They are located in close proximity to the stationary points $C_{1}$ and $C_{2}$.

An analysis of the behavior of the solutions of the equation depending on the thermodynamic parameters of the system [11] showed that the resonant state of interest to us is located in the vicinity of point $C_{2}$. It is in this interval of initial values for the initial equation that all the previously discussed conditions for the manifestation of the composition modulation effect in the solid solution, with the complex nature of oscillations, are realized. Indeed, an analysis of the position of the $C_{2}$ point at $450 \mathrm{~K}$ on the temperature - composition thermodynamic phase diagram [3-4] shows that the system is in close proximity to the spinodal of decay and falls into the region of the metastable state, which lies between the spinodal lines and the corresponding binodal. When the initial concentration is located slightly to the left of point $C_{2}$, the solution of the boundary problem shows a sharp increase in the amplitude of oscillations in the distribution of the composition over the layer thickness. This allows us to hope that in oscillations with such parameters, the nonlinear properties of differential eq. (1) will also be clearly manifested.

Thus, to get the unique solution of system (1) - (2), we constructed the iterative procedure for solving eq. (2) changing the initial data during the integration of system (1).
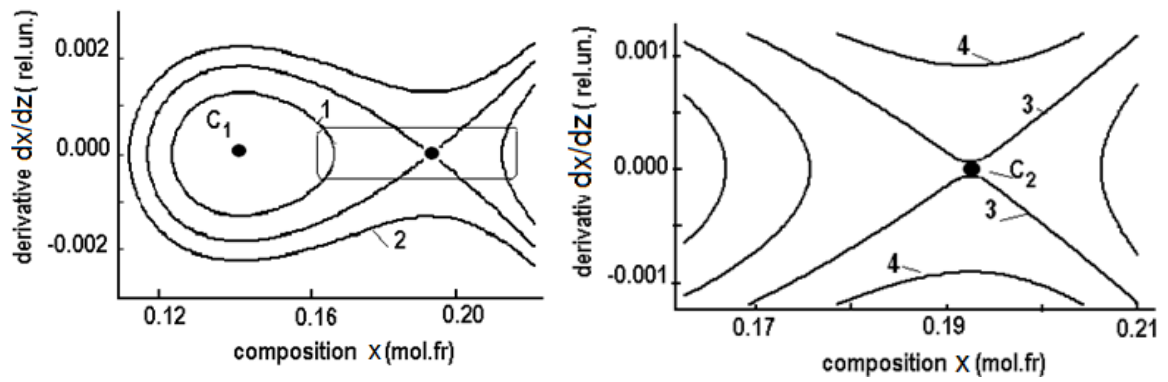

Fig.1. Dependence of the derivative $d x / d z$ on the composition for $Z n_{x} C d_{1-x}$ Te solid solutions at a temperature of $450 \mathrm{~K}$ (phase portrait), the orientation of the stabilizing substrate $\langle 111\rangle$, and $\mu=-190 \mathrm{~J} / \mathrm{mol}$. The points " $C_{1}$ and $C_{2}$ " mark the stationary points of differential eq. (1), i.e, concentration position of conditions $F\left(C_{1}\right)=0, F\left(C_{2}\right)=0$. Curve 1 corresponds to the total energy $E$ of the system [11] $E=0.5 \mathrm{~J} / \mathrm{mol}$;; $2-E=1.2$ $\mathrm{J} / \mathrm{mol}$;; $3-E=0.4 \mathrm{~J} / \mathrm{mol}$.; $4-E=0.8 \mathrm{~J} / \mathrm{mol}$. The right panel represents the enlargement of the rectangular domain in the left panel.

A set of testing and working calculation programs were implemented based on the standard "Mathematica" software package. This approach to software development allowed one to use proven standard procedures for solving differential and 
transcendental equations. This significantly reduced the total time spent on the development of mathematical and software to the problem.

The high efficiency of the developed algorithm is confirmed by the high rate of convergence and, accordingly, the low time spent on solving the problem. Typical machine time needed to find solutions to the original integro-differential problem was several minutes.

\section{Modelling the composition modulation effect during the spinodal decomposition of the $\mathrm{Zn}_{\mathrm{x}} \mathrm{Cd}_{1-\mathrm{x}}$ Tesolid solutions.}

The developed thermodynamic concepts of the spinodal decomposition of $\mathrm{Zn}_{\mathrm{x}} \mathrm{Cd}_{1-\mathrm{x}} \mathrm{Te}$ semiconductor solid solutions are applied to the description of the material decomposition process when the thermodynamic state of the material is stabilized by the elastic deformation of the initial crystal lattice. Unfortunately, there are no direct experimental results on the discovery of the composition modulation effect in this material during its synthesis under conditions when its state is close to the boundaries of spinodal decomposition. At the same time, a theoretically substantiated prediction of these material properties will be useful, both from the point of view of subsequent control of such processes and to obtain a material with predetermined electro-optical characteristics. The initial data for the modeling process and estimates of the model parameters will be experimental data on the study of the composition modulation effect in $\mathrm{A}^{3} \mathrm{~B}^{5}$ solid solutions [8-10]. It points out that the $\mathrm{Ga}_{\mathrm{x}} \operatorname{In}_{1-\mathrm{x}} \mathrm{P}, \mathrm{Ga}_{\mathrm{x}} \mathrm{In}_{1-\mathrm{x}} \mathrm{P}_{\mathrm{y}} \mathrm{As}_{1-\mathrm{y}}$ solid solutions are the close analogs for studying $\mathrm{Zn}_{\mathrm{x}} \mathrm{Cd}_{1-\mathrm{x}} \mathrm{Te}$ solid solution.

At the first stage of modeling, the substantiation of the parameters of the model was carried out, which would correspond to its physical meaning and ensure consistent forecasting of the parameters of the effects under consideration. As already noted, the fitting parameters of the model are the coefficient of power series for the free energy of a solid solution no uniform in composition $\beta$ and constants $\mu$ and $x_{\text {init. }}$ Moreover, the constant $\beta$, in essence, set only the period of composition oscillations in the solid solution. At the same time, the parameter $\mu$, which is interpreted from the physical point of view as the chemical potential of the system during spinodal decomposition, controls the amplitude of the oscillatory process. Naturally, the choice of the magnitude of these parameters was carried out in accordance with their physical meaning.

The parameter $\beta$ was estimated in [11], where experimental data on the oscillation period of the composition in $\mathrm{Ga}_{\mathrm{x}} \mathrm{In}_{1-\mathrm{x}} \mathrm{P}$ solid solution layers grown on a $\mathrm{GaAs}$ $<111>$ substrate from the intrinsic liquid phase were used [4]. Approximately the same data on the oscillation period was found for the decomposition of $\mathrm{Ga}_{\mathrm{x}} \mathrm{In}_{1-\mathrm{x}} \mathrm{P}_{\mathrm{y}} \mathrm{As}_{1-\mathrm{y}}$ solid solutions in a heterostructure formed on an InP substrate [9-10]. According to this data, the oscillation period of the composition of the quaternary solid solution is also at the level of $200-300 \mathrm{~nm}$. According to [11], the parameter $\beta$ is proportional to the square root of the oscillation period. That is, it is a relatively weak function of the magnitude of the input, initial experimental data. Therefore, based on the indicated mathematical fact and the fact that the periods of composition oscillations observed in experiments for related solid solutions are close in magnitude, in the further analysis, the parameter $\beta$ is taken close to the analogous one from [11]. To achieve agreement with the 
experimental data for the composition period oscillations of about $200 \mathrm{~nm}$, its value is taken equal to $\beta=2.4 \cdot 10^{5} \mathrm{~J} \mathrm{~nm}^{2} / \mathrm{mol}$.

As for the amplitude of the oscillatory process, in almost all cases, it was reported that the amplitude of the composition oscillations in $\mathrm{A}^{3} \mathrm{~B}^{5}$ solid solutions does not exceed 0.02 mol.fr. [8-10]. This range of the oscillatory process is naturally limited by the possibility of the existence of the coherent conjugation of inclusions of a new phase and the crystal lattice of the initial solution or, the same thing, of elastic stresses in the system. Indeed, higher oscillation amplitudes will inevitably violate the conditions for the existence of an elastically deformed phase conjugation. The latter will inevitably lead to a breakdown of the oscillatory process. This made it possible in the calculations to choose the parameter's value $\mu$, which would ensure the indicated oscillation amplitude.

A typical form of the composition distribution and CLP over the layer of $\mathrm{Zn}_{\mathrm{x}} \mathrm{Cd}_{1 \text { - }}$ xTe solid solution with $x_{\text {init }}=0.169$ mol.fr, which is synthesized at $T=450 \mathrm{~K}$ for a selected value of the parameter $\beta$, is shown in Figure 2. From this figure, it follows that the periodic structure of the distribution of the solid solution composition is visible in the direction of the growth of the layer. Moreover, the concentration profile of the components differs significantly from the shape corresponding to harmonic oscillations. This was a direct consequence of the complex dependence of the parameters of the differential equation for the distribution of concentrations on the composition of the material.

The calculated result of Figure 2 clearly illustrates the formation of concentration domains during the synthesis of the solid phase under thermodynamic conditions close to the boundaries of spinodal decomposition. Note that the values $\mu=-190 \mathrm{~J} / \mathrm{mol}$ and $x_{\text {init }}=0.191$ mol.fr. chosen in this calculation for $T=450 \mathrm{~K}$, are located on the abscissa of the phase portrait of eq. (1) (Figure 2) somewhat to the left of point $C_{2}$, i.e. lie on a curve describing the resonance between the energy causing the decomposition of the solid solution and the elastic energy created by the inclusions of the new final phase. This means that the solution found is in close proximity to the resonance state, which is characterized by a significant increase in the oscillation amplitude and, as a consequence, the manifestation of the nonlinear properties of the system.

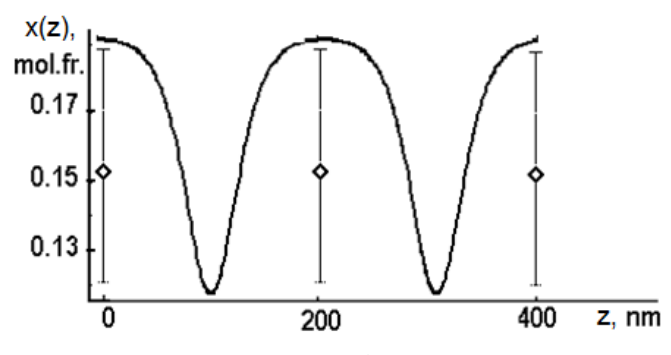

a) 


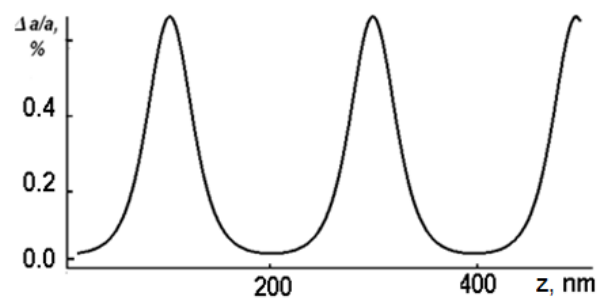

b)

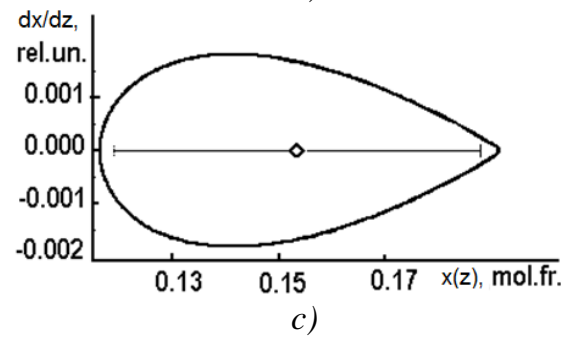

Fig. 2. Distribution of composition $x(z)(a)$ and of the relative lattice mismatch between CLP of new precipitates and CLP of the matrix of the starting material $\triangle a(z) / a(b)$ over the $\mathrm{Zn}_{x} C d_{1-x}$ Te solid solution layer (z-coordinate) synthesized at $T=450 \mathrm{~K}$ with an average composition $\bar{x}=0.198 \mathrm{~mol}$.fr. An experimental estimate of the oscillation amplitude was made according to data from [8-10]; c) Fragment of the phase portrait of the oscillation, providing the effect of modulation of the composition of the solid solution.

Figure 2 also presents generalized data on a typical value of the amplitude of composition oscillations, which were observed experimentally when studying the composition modulation effect in some $\mathrm{A}^{3} \mathrm{~B}^{5}$ solid solutions [8-10]. Figure 2 follows that, with the right choice of the parameter $\mu$, a quite satisfactory agreement between the calculations and the experiment can be achieved. It should be noted that such a correspondence to experiment is possible only with special relations between the values of mixing energy, elastic energy, and the chemical potential of the system. Indeed, if the maximum of the total energy is located in such a way that the points $C_{1}$ and $C_{2}$ are practically spaced relative to each other, then the amplitude of the oscillations will be very significant. Significant composition variations in such a process will lead to the appearance of large crystal lattice mismatches in coherently matching materials. In such a situation, the parameters of the system can exceed their critical values with the formation of dislocation networks and the breakdown of the coherent state of phases. The latter will inevitably lead to a breakdown of the oscillatory process, and in this case, the model under consideration is not applicable. The last argument allows us to make the following assumption. Due to the fact that for the composition modulation effect in the solid solution to occur, many factors must coincide, the effect under consideration should not be manifested in all cases when the growth system is in a state close to the spinodal decomposition. Therefore, the composition modulation effect should be 
recognized as an effect that should rarely occur in the practical implementation of the process of obtaining ternary solid solutions.

Figure 3 shows the results of modeling the effect of the spinodal decomposition of solid solutions at a temperature of their formation of $450 \mathrm{~K}$ for all material compositions. The calculated position of spinodal and binodal on the ternary $\mathrm{Zn}-\mathrm{Cd}-\mathrm{Te}$ state diagram with a known parameter $\alpha^{S}$ can be simply obtained using formulas, for example, from $[13,4]$.

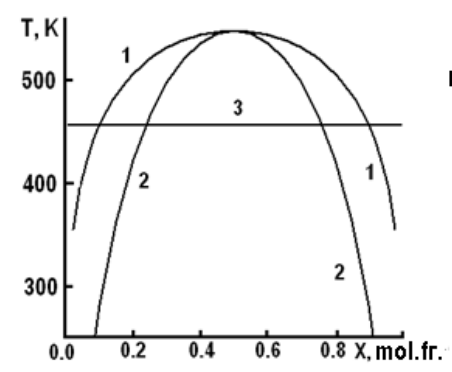

a)

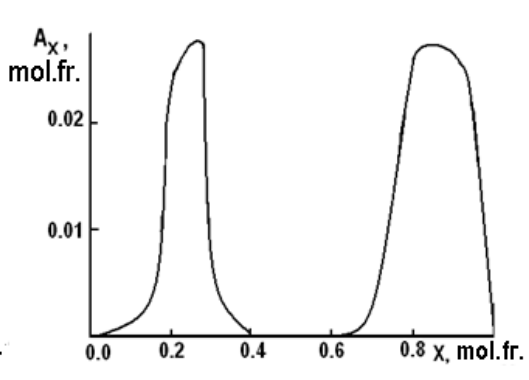

b)

Fig.3. The low-temperature fragment of the $\mathrm{Zn}$-Cd-Te phase diagram (a) and the dependence of the oscillation amplitude $A_{x}(b)$ arising at a material synthesis temperature of $450 \mathrm{~K}$ on the composition of the $\mathrm{Zn}_{x} \mathrm{Cd} d_{1-x} \mathrm{Te}$ solid solutions. Curves 1 and 2 are system's binodal and spinodal; 3 - isothermal cross section of the diagram at 450 $K$.

From the calculations, it follows that, as the composition of the solid solution approaches the boundaries of the stable and metastable region, the resonance conditions between the mixing energy and the energy of elastic deformations are fulfilled more and more accurately. This is reflected in an increase in the amplitude of composition oscillations of the solid solutions. With a significant removal of the thermodynamic state of the system from the boundaries of material instability, the resonance conditions are practically not satisfied. This means that there is no possibility for the development of intense oscillatory processes in the system, and therefore there is no composition modulation effect in the material. Therefore, it can be argued that the composition modulation effect in solid solutions may be intense and can be experimentally detected when the thermodynamic state of the system is very close to the boundaries of spinodal decomposition.

The appearance of periodic composition oscillations with a period of several nanometers should be taken into account when forming superthin layers or nanostructures based on $\mathrm{A}^{2} \mathrm{~B}^{6}$ solid solutions, when the thicknesses of the grown layers become comparable with the modulation period, and the material is synthesized at temperatures close to spinodal decomposition conditions. 


\section{Conclusion}

The adaptation of the Cahn-Hilliard equation is performed for describing the process of concentration domain formation at the spinodal decomposition of $\mathrm{Zn}_{\mathrm{x}} \mathrm{Cd}_{1-\mathrm{x}} \mathrm{Te}$ ternary solid solutions. This physical phenomenon is responsible for the appearance of micro oscillation of the composition of the solid solution (composition modulation effect) when it is synthesized under conditions close to a thermodynamically unstable state.

A quantitative description of the composition modulation effect is obtained by numerically solving the resulting differential equation. It is shown that the appearance of micro oscillations of the composition of the solid solution is associated with the resonance between the excess mixing energy of the decaying solid phase and the energy of elastic strains arising in the inclusions of the new solid phase, which are coherently conjugated with the crystal lattice of the initial material. The most favorable temperature and concentration conditions for the occurrence of such an oscillatory process are indicated on the state diagram of the system.

\section{References}

[1] P.P. Moskvin, V.B. Kryzhanivskyy, L.V. Rashkovetskyi, et al.: J Cryst Growth, 404 (2014) 204-209.

[2] P.P. Moskvin, V.B. Kryzhanivskyy, L.V. Rashkovetskyi, et al.: J Cryst Growth, 475 (2017) 144-149.

[3] A. Haloui, Y. Feutelais, B. Legendre: J Alloys and Compounds, 260 (1997) 179192.

[4] P.P. Moskvin, G. Olchowik, J.M. Olchowik: J Cryst Growth, 363 (2013) 195204.

[5] J.W. Cahn: Acta Metallurgica, 9 (1961) 81-87.

[6] J.W. Cahn, J.E. Hilliard: J. Chemical Physics, 28 (1958) 258-267.

[7] A.G. Khachaturyan (1983) Theory of Structural Transformations in Solid, Wiley, New York.

[8] S.K. Maximov, L.A. Bondarenko, V.V.Kuznetsov, A.S.Petrov: Soviet Phys Solid State, 24 (2) (1982) 628-631.

[9] P. Henoc, A. Izrael, M. Ouillec, H. Launois, Appl Phys Lett, 40 (1982) 963-965.

[10] M. Ouillec, H. Launois, M.S. Joncour: J Vac Sci Technol, 1 (1983) 238 - 242.

[11] P.P. Moskvin, S.I. Skurativskyi, O.P. Kravchenko et al: J Cryst Growth, 510 (2019) 40-46.

[12] V.V. Kuznetsov, P.P. Moskvinet et. al: J Phys: Conf Ser, 1697 (2020) 012092.

[13] P.P. Moskvin, L.V. Rashkovetskyi, F.F. Sizovetal: Semiconductors: Pleiades Publishing Ltd, 45 (2011) 837-844.

[14] I. Prigogine, R. Defay (1954) Chemical thermodynamics, Prentice Hall Press.

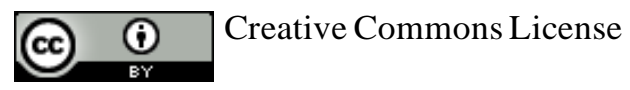

This work is licensed under a Creative Commons Attribution 4.0 International License. 